Article

\title{
Some Aspects of the Control for the Radial Distribution of Burden Material and Gas Flow in the Blast Furnace
}

\author{
Anatoliy Golovchenko ${ }^{1}{ }^{(0)}$, Roman Dychkovskyi ${ }^{2}{ }^{(D}$, Yuliya Pazynich $^{1}{ }^{(D}$, \\ Cáceres Cabana Edgar ${ }^{3}(\mathbb{D})$, Natalia Howaniec ${ }^{4}$ (D), Bartłomiej Jura ${ }^{5}$ and Adam Smolinski ${ }^{4, *(D)}$ \\ 1 Interdisciplinary Institute of Continuing Education, Dnipro University of Technology, \\ UA-49027 Dnipro, Ukraine; anatoliy@golovchenko.org (A.G.); jpazynich@ukr.net (Y.P.) \\ 2 Department of Development \& Research, Dnipro University of Technology, UA-49027 Dnipro, Ukraine; \\ dychkovskyi.r.o@nmu.one \\ 3 Scientific Research Institute of the Center of Renewable Energy and Energy Efficiency, Universidad Nacional \\ de San Agustin de Arequipa, Arequipa PE-04000, Peru; ecaceresca@unsa.edu.pe \\ 4 Department of Energy Saving and Air Protection, Central Mining Institute, 40-166 Katowice, Poland; \\ nhowaniec@gig.eu \\ 5 Experimental Mine Barbara, Central Mining Institute, Plac Gwarkow 1, 40-166 Katowice, Poland; \\ bjura@gig.eu \\ * Correspondence: smolin@gig.katowice.pl; Tel.: +48-32-259-2252
}

Received: 31 December 2019; Accepted: 17 February 2020; Published: 19 February 2020

\begin{abstract}
The paper presents an experimental study on the formation process of burden surface texture on the blast furnace throat and its influence on the radial distribution of gas flow. The study was performed with the application of blast furnaces equipped with a bell-type charging device using radio-isotope means for the control of burden surface texture (profile) and burden surface level, i.e., gamma locators for burden surface texture. The study was carried out under the conditions of an operating blast furnace in an iron and steel plant using a unique GEOTAPS system for automated control of geometric and temperature parameters of burden material surface on the blast furnace throat. The influence of the surface texture on the gas flow distribution was also investigated. The possibility of a self-stabilization effect for burden surface texture and gas flow in an operating blast furnace under suitable conditions was experimentally proven. As a result of the experimental study performed, four ways of energy-saving technology implementation were determined for the control of blast furnace melting based on the data on the burden surface texture and previously unknown regularities of surface layer formation of burden material on the throat of an operating blast furnace with a bell-type charging device. The main idea of the paper is the development of automated control for the radial distribution of burden material and gas flow using actual or predicted surface texture parameters as important intermediate factors that both describe the process and have a significant simultaneous influence on it.
\end{abstract}

Keywords: gas flow in blast furnace; energy saving; process efficiency

\section{Introduction}

Nowadays, clear trends of a significant increase in energy consumption are observed [1-4]. This is the reason why the issue of energy saving is of the highest priority in the energy policies of developed countries [5-8]. There exist national programs focused on the sustainable development of energy sectors as well as the sustainable use of energy resources [9-12]. 
Blast furnace ironmaking constitutes one of the most energy-consuming production processes. In this case, blast furnaces have specific types. The main product of melting (cast iron) is obtained as a result of burden material and gas flow interaction [13-16]. The creation of artificial shells for providing the dynamic processes was proved. The prepared steam for thermal influence on the anisotropic environment to change the metamorphic properties for the stability assurance of the gas flow was used in the research tests $[17,18]$. The complex thermal processes resulting from blast furnace melting were described by the Neumann principle. This principle was also used for the magnitude determination of the maximum stress vector (the polar tensor of the second rank) for the definition of the axial tensor of anisotropic mechanical deformations, which appeared under the high-temperature expansion. This process together was used as the base method for the definition of thermal destruction with detail given in [18]. There are also many other approaches to describe thermodynamic processes and the definition of models for representing the destructive effects on raw materials during blast furnace smelting that are available in the literature [16,19-23].

The burden material stepwise descends from the throat to the combustion region (lances). In terms of the gas-dynamic mode control, the simplest mode is the so-called unidimensional mode. In such a case, the surface of burden material on the blast furnace throat is horizontal, and the distribution of burden components and ascending gas flow in any horizontal section of the burden material column remains steady. Thus, the speed of material descent, as well as other technological parameters at any point of the section would be the same and changed only vertically. The creation of such an interaction mode for the gas flow and burden material is almost impossible under real conditions. The hopper angles of the surface may be changed by the gas flow in a wide range, namely from $0^{\circ}$ to values close to the natural burden material hopper. During the descent of the burden material column in the melting process, the typical discontinuity of different points of its horizontal sections occurs.

The solution to the problems of rational control of blast-furnace ironmaking requires considering the space discontinuity, transient effects, response time, transport delays, and the difficulty or even impossibility of operation control of some important process parameters. The control of burden material and gas flow on blast furnace throat is aimed at the decrease in energy consumption. It is achieved by automated stabilizing of the gas-dynamic mode of blast-furnace ironmaking. The most reasonable discontinuity in terms of an efficiency increase for gas energy use and the efficiency of the blast furnace is the discontinuity of gas distribution characterized by more intensive gas flow in relatively small areas of the central and peripheral zones of the throat. Developed central and peripheral gas flows provide the stability of the gas-dynamic mode of the blast furnace. At the same time, incorrect gas flows cause a decrease in the efficiency of gas energy use resulting in an increase in energy consumption.

The distribution of burden components with different permeability constitutes the main factor affecting the process of gas flow formation. Coke has the highest permeability, so the control of the radial distribution of gas flow is mainly provided by means of controlling the radial distribution of iron-bearing burden material in relation to coke (carbon-iron balance) as a result of the proper control of the charging device. The trough-type charging device has the greatest potential in terms of carbon-iron balance control.

The problem of controlling the radial distribution of burden material by means of a traditional method (based on gas radial distribution data), is that the control is highly delayed because of the creation of the proper structure of the burden column. It is the main factor in the gas flow formation process. The delay may result in low control efficiency of burden distribution in the case of an unstable gas dynamic mode, i.e., when the control is needed the most. The need for such a control increases with the reduction of specific coke consumption as an important factor of burden permeability and the stability of the gas dynamic mode of a blast furnace.

As for modern blast furnaces, the automated preventive control of radial burden distribution is based on mathematic modeling for the processes of burden column and gas flow formation, providing that the indicators of radial gas distribution are currently monitored and the maximum quantity of 
factors influencing the processes are considered. The parameters of burden surface texture on the blast furnace throat are of the utmost importance in terms of forecasting radial burden distribution among the other factors. This is proved by the results of many experimental studies involving physical models of a throat and operating blast furnaces by means of monitoring instruments for surface texture and column structure of the burden column.

The scientific problem of energy-saving control for the radial distribution of the burden and gas flow consists of the necessity of considering the high dynamics of the parameters change of burden surface texture. This is one of the most important factors that influence the distribution which justifies the limited possibilities for preventive control actions, especially considering the lack of current monitoring instruments for surface burden texture. The solution to this scientific problem is connected with complicated technical tasks of developing reliable instruments for continuing the monitoring of surface burden texture under operating conditions and the automated control of the radial distribution of the burden materials and gas flow.

The complexity of the problem results from the high level of dust content in the space above the burden, as well as the boiling and fluidizing of the burden surface layer caused by the gas flow [24-27]. The attempts at solving the problem by the application of mechanical, microwave and even x-ray monitoring instruments failed [28]. In particular, the experimental studies on $\mathrm{x}$-ray monitoring instruments under the conditions of the operating blast furnace at Magnitogorsk metallurgical plant have demonstrated the negative influence of small particles and dust moving upwards in the areas of the developed gas flow, especially in the throat axis zone, and thus mispresenting the results of surface texture control in the area. That is the reason why the gamma-ray sources are almost the only possibility of reliable monitoring of the burden surface texture under operating conditions because of their high penetration properties. The fact is confirmed by the experimental operating of radio-isotopic means of control for the burden surface texture (gamma locators) in Ukraine and abroad using cobalt-60 and cesium-137 as well as other isotopes [29]. At the same time, the development and application of gamma locators in blast furnaces are restrained, mainly because of their radiological danger.

The main objective of the paper is to develop scientific and technical preconditions for the development of an energy-saving control technology for radial distribution of the burden and gas flow in blast furnaces by:

- The development of radio-isotopic means for current control of burden surface texture on the throat of an operating blast furnace and the determination of its technical capacity;

- The substantiation of general parameters of burden surface texture under operating conditions;

- The study of the interrelations between general parameters of burden surface texture and the main parameters of blast furnace ironmaking;

- The development of recommendations for considering the parameters of burden surface texture in the control system for the radial distribution of the burden and gas flow.

\section{Materials and Methods}

For the purposes of control automatization of the radial distribution of burden using the parameters of burden surface texture, it is essential that the texture is described by a minimum of generalized indicators. In order to meet this condition, we substantiate two indicators-volume $V_{B}$ (or depth) of a hopper on the burden surface and an average level of a surface. The calculations of the indicators are closely related to the distribution of burden and gas flow and may be carried out using the following formula:

$$
\begin{gathered}
V_{B}=\sum_{j=1}^{J}\left(\sum_{i=1}^{I_{j}} \Delta V_{j, i}\right), \\
H_{m}=\frac{4 V_{B}}{\pi D_{K}^{2}}+\frac{1}{J} \sum_{j=1}^{J} h_{j, 1},
\end{gathered}
$$


where

$J=$ stands for the number of radii (segments) of the throat;

$I_{j}=$ stands for the number of texture points of the surface in $j$-segment, the first being in the periphery, the latter, in the center of the throat;

$\Delta V_{j, i}=\frac{\pi}{3 j}\left(h_{j, i+1}-h_{j, i}\right)\left(r_{j, i}^{2}+r_{j, i} r_{j, i+1}+r_{j, i+1}^{2}\right)=$ stands for volume $j$, of $i$-part of a hopper considered as a part of a truncated cone between $i$-point and $(I+1)$-point of a surface profile in $j$-segment;

$h_{j, i}=$ stands for $y$ coordinate of $i$-point of j-profile;

$r_{j, i}=$ stands for $i$-point distance of $j$-profile form a throat axis;

$D_{K}=$ stands for a throat diameter.

The study of the interrelations between general parameters of burden surface texture and the process of the surface texture formation in an operating blast furnace was performed by means of active and passive experiments in blast furnace $\# 1$, volume $1386 \mathrm{~m}^{3}$, and \#3, volume $2002 \mathrm{~m}^{3}$. The burden surface profiles were monitored according to one or two mutually perpendicular throat diameters by means of radio-isotopic control for burden surface texture [30]. Each measuring cycle involved consistent monitoring of three profiles: after regular burden load, in approximately $5 \mathrm{~min}$, and straight after the next load. This enabled obtaining all the necessary information on the dynamics of burden surface changes.

In these studies, as the basis of the blast furnace melting and control of the production process, the known principles of the compliance with the ratio of ore volume to coke volume were used. Accordingly, the accepted classification shows the order of the coke (C) and the agglomerate (A) loading, and the arrow indicates the lowering of the cone:

- $\quad$ CCAA $\downarrow$-reverse feed, first coke then agglomerate;

- $\quad$ CACA $\downarrow$-reverse alternating feed in the system: coke-agglomerate; coke-agglomerate;

- $\quad$ CAAC $\downarrow$-so-called feed: one coke forward;

- $\quad \mathrm{CA} \downarrow \mathrm{CA} \downarrow$-reverse two-stage feed: coke—agglomerate; coke-agglomerate;

- $\quad \mathrm{AACC} \downarrow$ - direct feed, first agglomerate then coke;

- $\quad A C A C \downarrow-$ direct variable feed: agglomerate—coke; agglomerate-coke;

- $\mathrm{AC} \downarrow \mathrm{AC} \downarrow$ - direct two-stage feed: agglomerate—coke; agglomerate—coke.

The one- and two-stage process is determined by the amount of lowering of the cone.

On the basis of data analysis from a series of passive experiments during some weeks, the model was substantiated for the process of radial burden and gas flow distribution. The volume of a hopper $V_{B}=Z_{1}$ on the surface was considered as an intermediate factor of the process. The level of burden charge $X_{1}$, mass $X_{2}$, the relative quantity of pellets in burden $X_{3}$, the indicator of charging system $X_{4}$, and the level of central gas flow development $Z_{3}$ were set as the main factors influencing the process of hopper formation (block 1 in Figure 1). In order to determine the quantitative characteristics of the charging system $X_{4}$, published data on its influence on the radial burden and gas flow distribution were used. The value of $X_{4}$ indicator was determined by ranging the used charging system with respect to its influence on peripheral gas flow (CCAA $\downarrow, C A C A \downarrow, C A A C \downarrow, C A \downarrow C A \downarrow, A C A C \downarrow, A A C C \downarrow$, $\mathrm{AC} \downarrow \mathrm{AC} \downarrow \downarrow)$, and providing the $X_{4}$ indicator with the proper order number in the row. The level of the central gas flow development $Z_{3}$ was determined as the relation of maximum carbon dioxide content in the gas and its content in the throat central zone. The volume of the funnel significantly affects the ore load on coke in the central area of the grate $Z_{2}$ (block 2 in Figure 1) and the degree of the central gas stream in the blast furnace $Z_{3}$ (block 3 in Figure 1). 


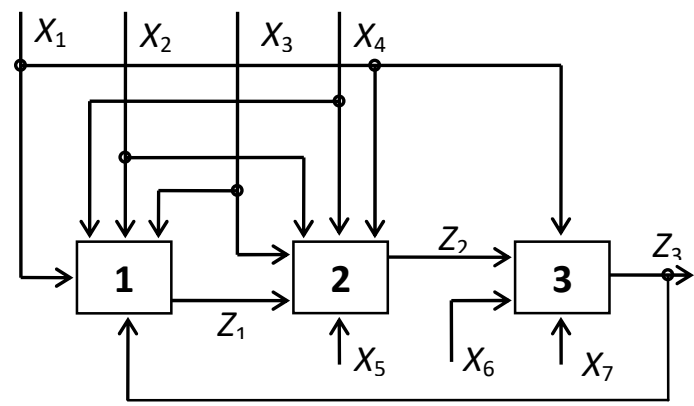

Figure 1. Model of the process of the burden and the gas flow radial distribution.

The following main factors are taken into account:

- $\quad X_{1}$-the charge backfills level (m);

- $X_{2}$-the mass of the feed at each lowering of the large cone $(t)$;

- $X_{3}$-the relative number of pellets in the charge $(\%)$, and/or ore load ( $t$ ore/t coke, average content in the charge);

- $X_{4}$-an index of filling and assign it the appropriate system sequence number;

- $X_{5}$-ore load in the process of charge descent (mass of ore per $t$ of cast iron);

- $\mathrm{X}_{6}$-the degree of filling of blast furnace products (\%);

- $X_{7}$-kinetic energy of the blast $(\mathrm{kgf} \cdot \mathrm{m} / \mathrm{s})$.

The process of the burden column formation (Figure 1) occurs progressively influenced by the factors already published and ore load $X_{5}$ during the burden descent and its charge [29]. This process, which may be described in terms of ore load $Z_{2}$ in the throat central zone, the level of smelting by-products content in a blast furnace $X_{6}$ and kinetic blast energy $X_{7}$ have the greatest influence on the process of the central gas flow formation (block 3 in Figure 1). The summarized parameters of the main technological locks for burden column formation are presented in Table 1.

Table 1. The parameters of the main technological locks for burden column formation.

\begin{tabular}{|c|c|c|c|c|}
\hline \multirow{2}{*}{ \# } & \multirow{2}{*}{ Parameters of the Main Technological Blocks } & \multicolumn{3}{|c|}{ Parameter Value } \\
\hline & & Minimum & Medium & Maximum \\
\hline 1 & $\mathrm{Z}_{1}, \mathrm{~m}^{3}$ & 1.41 & 1.92 & 2.42 \\
\hline 2 & $Z_{2}$, (ore load, $t$ ore/t coke, in the central area of the grate) & 3.69 & 3.75 & 3.81 \\
\hline 3 & $Z_{3}$, (dimensionless value) & 0.92 & 0.92 & 0.92 \\
\hline 4 & $X_{1}, \mathrm{~m}$ & 0.55 & 0.61 & 0.66 \\
\hline 5 & $X_{2}, \mathrm{t}$ & 101 & 106 & 111 \\
\hline 6 & $X_{3}, \%$ (ore load, t ore/t coke, average content) & $45.9(3.61)$ & $46.5(3.71)$ & $47.1(3.81)$ \\
\hline 7 & $X_{4},($ dimensionless value $)$ & $1-7$ & $8-14$ & $15-21$ \\
\hline 8 & $X_{5}$ (mass of ore per ton of iron) & 0.83 & 0.85 & 0.87 \\
\hline 9 & $X_{6}, \%$ & 0.86 & 0.87 & 0.88 \\
\hline 10 & $X_{7}, \mathrm{kgf} \cdot \mathrm{m} / \mathrm{s}$ & 2260 & 2635 & 3010 \\
\hline
\end{tabular}

It was found that the hopper volume, or depth, is changed without observable delay, the change is proportional to the development of the central gas flow in the case when other factors influencing the process of burden surface formation remain steady. The hopper volume also provides pressure difference reduction in the top zone of a blast furnace, as well as the stabilization of blast furnace ironmaking. Extra growth of hopper depth is possible based on the increase in relative pellets quantity in burden ore components, the reduction of burden drop height, and the mass of charge. 
For choosing the mathematical model of processes in the blast furnace, the entire complexity of geo-mechanical, heating, and technological factors should be taken into account. In the process of heat exchange, both the temperature field as well as the area of thermal action on the charge may vary. Temperature dependence of physico-mechanical characteristics changes in the temperature field within a zone of thermal effect and will result in the formation of the area where these characteristics experience non-uniform distribution. Moreover, temperature deformations will arise within the thermal action resulting from the thermal expansion. Taken together, the factors become the basis of forming a thermo-stressed state within a boundary of the charge in the blast furnace. Analysis of earlier studies has shown that the listed features of geo-mechanical processes may be considered adequate only within a continual model of nonhomogeneous mass. Thus, the studies use a spatial continual nonlinear model as the calculation procedure. According to the model, the charge is simulated as an elastic-plastic continuum with a cavity having a fritted shell. Stress-strain state and the cavity shell are described with the help of deformation plasticity theory. After their transformation, the deformation theory of thermal plasticity may be expressed through Duhamel-Neumann law $[17,18]$.

\section{Results and Discussion}

\subsection{Study of Interrelations between General Parameters of Burden Surface Texture and Main Parameters of Blast Furnace Ironmaking}

In order to determine the optimum hopper depth on the burden surface, a correlative analysis was conducted on the basis of the data from passive experiments on blast furnace \#1. The analysis was based on the abovementioned peculiarities of the process of the radial distribution of the burden and gas flow. It also took into account the commonly known extreme influence of the development degree of the central gas flow on the main technical and economic indicators of blast furnace ironmaking. While determining the optimum hopper parameters, the conditions of maximum performance and blast furnace heating were considered as optimality criteria with respect to the possibility of reducing specific coke consumption in case such conditions are created. The degree of the blast furnace heating was estimated according to the relative silicon content in cast iron. As a result of a correlative analysis using the optimality criterion, it was found that the optimum hopper has a depth within $0.14-0.2$ of the throat diameter. The delay of the blast furnace performance is $6 \mathrm{~h}$; the influence of the blast furnace heating process is $13 \mathrm{~h}$. The profiles with such a hopper are formed in the case of a sufficient penetration of the burden column and ordinary gas flow in the central zone.

The experimental studies on the process of burden surface formation on the throat of an operating blast furnace with a bell-type charging device enabled us to observe an unknown regularity of the process. The regularity consists of the fact that during the charging, the surface brace angles in the charge zone are changed in an inverse proportion to the development degree of the rising gas flow in the zone, and after charging, the angles and burden surface texture are almost unchanged during the burden column descent. The formation of the particular texture of the burden surface mainly occurs at the moment of charging a definite mass of burden with the occurrence of a fluidizing phenomenon of the burden surface top layer by the rising gas flow. The extreme form of the effect being observed under the operating conditions using radio-isotopic means of control for the burden texture is shown in Figure 2.

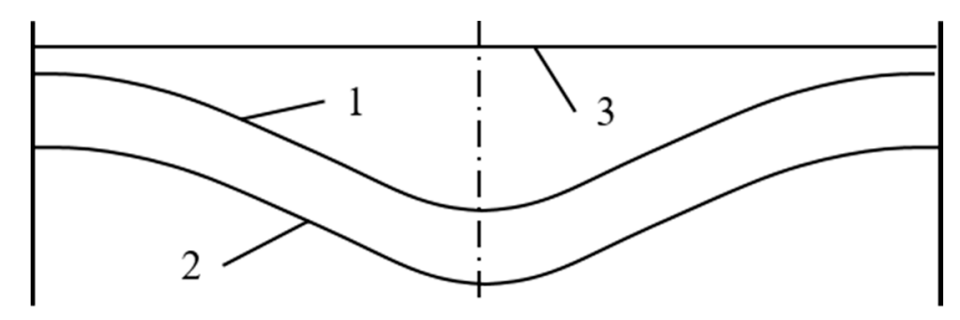

Figure 2. The extreme form of the fluidizing effect during the burden surface formation. 
Profiles 1 and 2 were recorded in the period of some minutes within the process of the burden descent for the ordinary development of the central gas flow, profile 3 was recorded after the burden charge to surface 2 . Thus, the results obtained are different from the known concepts concerning the regularities of burden surface formation.

3.2. The Development of Recommendations for Considering the Parameters of the Burden Surface Texture in the Control System for the Radial Distribution of the Burden and the Gas Flow

Gamma profilometers were developed for controlling the radial distribution of burden material using parameters of the burden surface texture. The first experiments were carried out on the blast furnace with the capacity of $1386 \mathrm{~m}^{3}$. They were targeted at practicing the technological methods of hopper depth stabilization on the burden surface within an optimal range. The possibility and efficiency of hopper depth control were demonstrated in accordance with a new method of blast furnace ironmaking. The control of hopper depth is based on the proportional ratio and the development level of the central gas flow by applying common control methods of radial gas distribution. It was found that the simple hopper control iobtained for its depth is more than 0.13 of throat diameter. The hopper control becomes complicated for a depth of less than 0.08 of throat diameter. The implementation of the developed manual enabled a $0.5 \%$ improvement of blast furnace performance and $2 \mathrm{~kg}$ reduction of coke consumption per ton of cast iron.

The second series of experiments were performed on two blast furnaces of $1386 \mathrm{~m}^{3}$ and $2002 \mathrm{~m}^{3}$. The experiments were focused on examining the conditions of the hopper self-stabilization effect on the burden surface and the central gas flow in the case of using the so-called direct sessions of AACC $\downarrow$ (combined flow: A agglomerate, A agglomerate, $C$ coke, $C$ coke) in the blast furnace charge system. The possibility of such an effect is substantiated by the proportional ratio of hopper depth or volume $Z_{1}$ to the parameters of the central gas flow $Z_{3}$, the proportional ratio of ore load $Z_{2}$ in the central zone to $Z_{1}$ in the case of direct charge use, as well as common inverse proportional ratio of $Z_{3}$ and $Z_{2}$ (Figure 3). The additional simultaneous control of the coke ore load in the peripheral zone by means of gammas absorption index monitoring for the surface burden layer was carried out using the method described in the paper [30]. As a result of the experiments, it was found that the effect of hopper self-stabilization and the central gas flow occurs only in the case of a high permeability of the burden material column. Figure 3 demonstrates the framework of the effect, where the processes of hopper formation on the burden surface, the radial distribution of the burden, and the gas flow are considered using proper line structure elements with speed ratios $K_{Z 1}, K_{Z 2}$, and $K_{Z 3}$ [31].

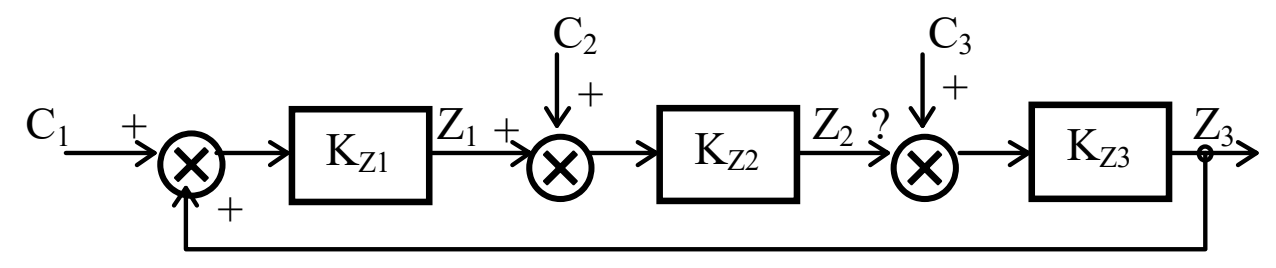

Figure 3. The framework of hopper self-stabilization, the radial distribution of the burden and gas flow.

Factors $X_{1} \ldots X_{4}$ on $Z_{1}, X_{1} \ldots X_{5}$ on $Z_{2}$ and $X_{1}, X_{6}, X_{7}$ on $Z_{3}$ in Figure 3 are considered relatively by direct generalizing signals $C_{1}, C_{2}$, and $C_{3}$, respectively. They may be determined by the multiple regression equation for $Z_{3}$, as a function of controlled factors $X_{1} \ldots X_{7}$ and $Z_{1}$. The self-stabilization effect is violated in the case of insufficient permeability of the burden column material represented by the reduction of the influence of direct charges AACC $\downarrow$ on the development of the central gas flow, provided that the depth of the hopper is relatively small. In such a case, the ore load on the periphery increases significantly, which is proved by the monitoring results of the gammas absorption index by surface burden level; the pressure difference also increases greatly at the top of the blast furnace. It was found that the main destabilizing factors are as follows: the worsening of burden grain fineness was the main indicator considered as the relative pellets quantity in the ore part of the burden, a level too 
low of burden, the high level of blast furnace heating, an insufficient kinetic blast energy, as well as the use of direct charge during the period of stable hopper at a depth of less than 0.08 throat diameter for more than $30 \mathrm{~min}$.

On the base of the self-stabilization effect, two exploratory prototypes of a monitoring and control system for the blast furnace charge mode were developed. The system consisted of a gamma profilometer as a technological blast furnace control and measuring instrument with the system of twenty-four-hour recording of the generalized indicators of the burden surface texture and recommendations for the indicators (second, an improved variant of energy-saving technology for blast furnace ironmaking control). The use of the maximum possible quantity of the most efficient direct charges AACC $\downarrow$ in the case of a timely turn to CAAC $\downarrow$ charge type under the conditions of the stable (for more than $30 \mathrm{~min}$ ) existence of a hopper on the burden surface with the depth of no less than 0.08 throat diameter.

It was found that the exploratory prototypes of the control system for the charge mode provided an efficiency increase for the blast furnace and an average 2-3\% reduction of specific coke consumption. It was also recommended that the system operation was continued in blast furnaces to obtain needed data. The control system was advantageous to be implemented in blast furnaces with trough charge equipment. A proper technical design specification was developed and approved for the development of exploratory prototypes of gamma profilometers to be used in ACS for the charge mode in accordance with the proposed recommendations and control algorithms. The manufacturing of exploratory prototypes of the RIAP type was established.

In order to implement the developed control system in blast furnaces with trough charge equipment, a computer system GEOTAPS was developed for gamma and infrared monitoring of the burden surface. It consisted of an exploratory prototype of a gamma profilometer as well as recommendations for the determination and use of generalizing indicators of the burden surface texture in the control of the radial distribution of the burden and the gas flow [32,33]. Considering a separate charge of burden components in blast furnaces with trough charge equipment, the GEOTAPS system provides the possibility of determining and demonstrating the radial distribution of ore material and coke being the main factors influencing the distribution and efficiency of gas flow energy use.

The GEOTAPS system was implemented in blast furnace \#2, whose volume equals $1613 \mathrm{~m}^{3}$, of SSAB iron and steel plant trough-type charging device (the third variant of energy-saving technology for blast furnace iron making control). The comprehensive informational content of the developed system enabled deepening knowledge in the field of the main regularities of burden surface formation on a blast furnace throat with trough charge equipment and significantly reduced specific coke consumption. In particular, the previously unknown significant coke shift into the central zone was discovered. The phenomenon is caused by the kinetic influence of ore material during the charge and by the fluidizing effect of the burden surface layer resulting from the rising gas flow. The following data were considered:

- Predicted radial distribution of the known volume of each material charge (coke and ore material, respectively) in accordance with the existing parameters of trough charge equipment;

- The results of the gamma control of geometric parameters (texture) of coke surface and ore material surface, respectively, as well as their change dynamics (provided by means of measuring two surface profiles some minutes apart during the burden descent process);

- Actual geometric parameters of the burden surface before charging and after charging the mass of coke or ore material with respect to the dynamics of the burden descent and the differences in gammas penetration into coke and ore material (the profile of coke surface is shifted down for approximately $70 \mathrm{~mm}$ more than ore material because of deeper penetration of gammas into coke);

- The results of the infrared temperature monitoring of the burden material surface by means of the GEOMET 800 infrared imaging system being a part of GEOTAPS. 
Some experiments were conducted in blast furnaces for the purpose of studying the possibilities of using the self-stabilization effect of the burden surface texture in the case of a lack of direct monitoring means. The indicators of radial gas distribution were used as the intermediated data of the burden surface texture. Based on the studies, it was found that there is a possibility of a significant efficiency increase for the existing systems of the charge and radial burden distribution without using direct monitoring means of the burden surface texture. This was obtained as a result of applying the principle of preventive control actions in the case of the change of factors influencing the surface texture. In some periods of up to two weeks, it was possible to increase the number of direct charges in the charging system to almost $100 \%$ with the stability of the gas-dynamic mode of the blast furnace (the fourth variant of the energy-saving technology for blast furnace ironmaking control). In such periods, the reduction of specific coke consumption and blast furnace performance reached $5.7 \%$, which means significant untapped reserves. The inadmissibility of using direct charges in the case of an essential equipment failure in the blast furnace charging system was proved.

In further studies, the scope of the experiments is planned to be widened to change the volume ratios of coke and agglomerate, as well as to confirm the existing analytical calculations of the practical implementation of the charge self-stabilization in the blast furnace. The importance of the experiments consists of the verification of efficiency improvements for the existing control systems of the radial burden distribution without the use of radioactive hazardous monitoring of the burden surface texture. The maximum efficiency of the fourth variant of energy-saving technology for blast furnace ironmaking control occurs in the case of a significant reduction of natural gas consumption, which constitutes an additional energy-saving factor.

\section{Conclusions}

The paper presents the experimental research work related to the process of formation of the burden surface texture in the blast furnace throat by means of a particular charging device and an advanced system for automated control of the geometry and the temperature of the burden surface itself. It contains the control of the radial distribution of burden materials on a blast furnace throat based on the automated gamma monitoring of its surface. The solution to the problem is based on the following results:

1. The formation process of the burden surface level and the gas flow on a blast furnace throat were studied. It was proved that the volume or depth of a hopper on the burden surface is the main generalizing indicator influencing significantly the radial burden distribution and it is change in proportion to the gas flow development almost without delay.

2. The correlation analysis of the experimental results enabled determining the optimum depth of hopper on the burden surface within $0.14-0.2$ of the throat diameter, as well as substantiating its stabilization methods in the optimum range using common control methods of radial gas flow distribution. The values are observed in the case of the best performance of the blast furnace. The possibility of stabilization is limited in the period of stable hopper depth being less than 0.08 of throat diameter.

3. The possibility of the self-stabilization effect for the burden surface texture and the gas flow in an operating blast furnace with a bell-type charging device was experimentally proved for the first time in the case of using an energy-saving charge system; in addition, the conditions in which the effect occurs were determined. The previously unknown regularity of burden surface formation on the throat of the operating blast furnace was found. It consists of the fact that the hopper depth on the surface is mainly changed during the charging process rather than during the burden material column descend after charging.

4. The radio-isotopic means of current control for the burden surface texture on the blast furnace throat were proposed for the first time. They allow the timely formation of control actions for gas dynamics stabilization of blast furnace ironmaking. A range of energy-saving ways and variants of their implementation are substantiated. They consist of the use of the self-stabilization effect of 
the hopper depth and the gas flow. The methods imply using direct charges in the blast furnace charge system and a timely refusal from the direct charge in the case of hopper existence with the depth of 0.08 throat diameter for more than $30 \mathrm{~min}$.

5. The practical possibility for current simultaneous monitoring of geometric and temperature parameters of the burden material surface by means of a unique monitoring system GEOTAPS using gamma and infrared radiation was demonstrated.

6. The recommendations on efficiency improvements for the existing control systems of the radial distribution of the burden and the gas flow in the case of the deficiency of direct monitoring instruments for the surface burden texture were developed and proved experimentally. The control efficiency is increased as a result of applying radial distribution indicators of the gas flow in the blast furnace as the intermediated data of the burden surface texture and using the self-stabilization effect of the hopper depth on the surface.

Author Contributions: Conceptualization: A.G., R.D., Y.P., C.C.E., N.H., B.J. and A.S.; methodology: A.G., R.D., Y.P., C.C.E., N.H., B.J. and A.S.; formal analysis, A.G., R.D.; investigation, A.G., R.D., Y.P.; writing-original draft preparation, A.G., R.D., C.C.E., N.H., B.J.; writing-review and editing, N.H.; visualization, A.G., R.D., C.C.E.; supervision: A.S. All authors have read and agree to the published version of the manuscript.

Funding: This research received no external funding.

Conflicts of Interest: The authors declare no conflict of interest.

\section{References}

1. Medunić, G.; Mondol, D.; Rađenović, A.; Nazir, S. Review of the latest research on coal, environment, and clean technologies. Rud. Geol. Naft. Zb. 2018, 33, 13-21. [CrossRef]

2. Iwaszenko, S.; Howaniec, N.; Smoliński, A. Determination of random pore model parameters for underground coal gasification simulation. Energy 2019, 166, 972-978. [CrossRef]

3. Wojtacha-Rychter, K.; Smolinski, A. A study of dynamic adsorption of propylene and ethylene emitted from the process of coal self-heating. Sci. Rep. 2019, 9, 18277. [CrossRef] [PubMed]

4. Howaniec, N.; Smoliński, A. Biowaste utilization in the process of co-gasification with hard coal and lignite. Energy 2017, 118, 18-23. [CrossRef]

5. Rozin, V.M. Technology as a time challenge: Study, concept and types of technology. Philos. Cosmol. 2017, 19, 133-142.

6. Basu, R. Evaluation of some renewable energy technologies. Min. Min. Depos. 2017, 11, 29-37. [CrossRef]

7. Smil, V. A new world of energy. Camb. World Hist. 2015, 7, 164-184.

8. Nosić, A.; Karasalihović Sedlar, D.; Jukić, L. Oil and gas futures and options market. Rud. Geol. Naft. Zb. 2017, 32, 45-54. [CrossRef]

9. Beshta, O.S. Electric drives adjustment for improvement of energy efficiency of technological processes. Nauk. Visnyk Natsionalnoho Hirnychoho Universytetu 2012, 4, 98-107.

10. Pivnyak, G.; Razumny, Y.; Zaika, V. The problems of power supply and power saving in the mining industry of Ukraine. Arch. Min. Sci. 2009, 54, 5-12.

11. Vorob'ev, A.; Chekushina, T.; Vorob'ev, K. Russian national technological initiative in the sphere of mineral resource usage. Rud. Geol. Naft. Zb. 2017, 32,1-8. [CrossRef]

12. Pazynich, Y.; Kolb, A.; Potempa, M. Implementation of energy safety policy in Ukraine by means of energy saving in electric drive systems. Adv. Eng. Forum 2017, 25, 96-105. [CrossRef]

13. Voloshyn, O.; Potapchuk, I.; Zhevzhyk, O.; Yemelianenko, V.; Horiachkin, V.; Zhovtonoha, M.; Semenenko Ye Tatarko, L. Study of the plasma flow interaction with the borehole surface in the process of its thermal reaming. Min. Min. Depos. 2018, 12, 28-35. [CrossRef]

14. Xin, L.; Wang, Z.; Huang, W.; Kang, G.; Lu, X.; Zhang, P.; Wang, J. Temperature field distribution of burnt surrounding rock in UCG stope. Int. J. Min. Sci. Technol. 2014, 24, 573-580. [CrossRef]

15. Luo, J.; Wang, L.; Tang, F.; He, Y.; Zheng, L. Variation in the temperature field of rocks overlying a high-temperature cavity during underground coal gasification. Min. Sci. Technol. 2011, 21, 709-713. [CrossRef] 
16. Sobolev, V.V.; Usherenko, S.M. Shock-wave initiation of nuclear transmutation of chemical elements. J. De Phys. IV 2006, 134, 977-982. [CrossRef]

17. Dychkovskyi, R.O. Forming the bilayer artificial shell of georeactor in underground coal gasification. Nauk. Visnyk Natsionalnoho Hirnychoho Universytetu 2015, 5, 37-42.

18. Pivnyak, G.; Dychkovskyi, R.; Bobyliov, O.; Cabana, C.E.; Smoliński, A. Mathematical and geomechanical model in physical and chemical processes of underground coal gasification. Solid State Phenom. 2018, 277, 1-16. [CrossRef]

19. Lampert, K.; Ziebik, A.; Stanek, W. Thermoeconomical analysis of $\mathrm{CO}_{2}$ removal from the Corex export gas and its integration with the blast-furnace assembly and metallurgical combined heat and power (CHP) plant. Energy 2010, 35, 1188-1195. [CrossRef]

20. Shashenko, A.; Gapieiev, S.; Solodyankin, A. Numerical simulation of the elastic-plastic state of rock mass around horizontal workings. Arch. Min. Sci. 2009, 54, 341-348.

21. Riva, L.; Surup, G.R. Therese Videm Buo \& Henrik Kofoed Nielsen. A study of densified biochar as carbon source in the silicon and ferrosilicon production. Energy 2019, 181, 985-996. [CrossRef]

22. Mikhlin, Y.V.; Zhupiev, A.L. An application of the ince algebraization to the stability of non-linear normal vibration modes. Int. J. Non-Linear Mech. 1997, 32, 393-409. [CrossRef]

23. Sarycheva, L. Using GMDH in ecological and socio-economical monitoring problems. Syst. Anal. Model. Simul. 2003, 43, 1409-1414. [CrossRef]

24. Asanov, A.; Mekenbaev, B.; Chalybekov, D.; Arziev, J. Study of the process of coal transfer by means of auger effector within the aggregate of boiling bed. Min. Min. Depos. 2017, 11, 79-85. [CrossRef]

25. Falshtynskyi, V.S. Formation of thermal fields by the energy-chemical complex of coal gasification. Nauk. Visnyk Natsionalnoho Hirnychoho Universytetu 2017, 5, 36-42.

26. Tabachenko, M. Features of setting up a complex, combined and zero-waste gasifier plant. Min. Min. Depos. 2016, 10, 37-45. [CrossRef]

27. Dychkovskyi, R.; Vladyko, O.; Maltsev, D.; Cáceres Cabana, E. Some aspects of the compatibility of mineral mining technologies. Rud. Geol. Naft. Zb. 2018, 42, 73-82. [CrossRef]

28. Chernai, A.V.; Sobolev, V.V.; Chernai, V.A.; Ilyushin, M.A.; Dlugashek, A. Laser ignition of explosive compositions based on di-(3-hydrazino-4-amino-1,2,3-triazole)-copper(II) perchlorate. Combust. Explos. Shock Waves 2003, 39, 335-339. [CrossRef]

29. Golovchenko, A.; Pazynich, Y.; Potempa, M. Automated Monitoring of Physical Processes of Formation of Burden Material Surface and Gas Flow in Blast Furnace. Solid State Phenom. 2018, 277, 54-65. [CrossRef]

30. Golovchenko, A.S. The Device for Determination of Level and Profile of Materials Surface. Ukraine Patent No. 31479, 15 December 2000.

31. Golovchenko, A.S. The Way of Blast Furnace Melting Conducting. Ukraine Patent No. 31478, 15 December 2000.

32. Pivnyak, G.; Dychkovskyi, R.; Smirnov, A.; Cherednichenko, Y. Some aspects on the software simulation implementation in thin coal seams mining. Energy Effic. Improv. Geotech. Syst. 2013,1-10. [CrossRef]

33. Dychkovskyi, R.O. Determination of the rock subsidence spacing in the well underground coal gasification. Naukovyi Visnyk Natsionalnoho Hirnychoho Universytetu 2015, 6, 30-36.

(C) 2020 by the authors. Licensee MDPI, Basel, Switzerland. This article is an open access article distributed under the terms and conditions of the Creative Commons Attribution (CC BY) license (http://creativecommons.org/licenses/by/4.0/). 